Jean-Luc Egger

\title{
Intorno al «Normkonzept»: la traduzione italiana del «Gesetzgebungsleitfaden»
}

La pubblicazione della prima edizione italiana della «Guida di legislazione» offre lo spunto per alcune riflessioni sulle implicazioni concettuali, operative ed istituzionali della prassi traduttiva, ma anche sui vantaggi del plurilinguismo.

Categoria di articoli: Resoconti dell'attività

Citazione: Jean-Luc Egger, Intorno al «Normkonzept»: la traduzione italiana del «Gesetzgebungsleitfaden», in: LeGes 31 (2020) 1 
[1] Tradurre non è mai un atto innocente. Secondo alcuni studiosi l'equivalente che i latini, segnatamente CiCERONE, proposero per tradurre la parola greca nomos, cioè lex, fu fatale per tutta la successiva comprensione del fenomeno giuridico in senso lato, giacché il termine legge, che da lex deriva, occulta la dimensione non istituita, non positiva, l'ordine spontaneo, contenuta nel concetto di nomos, ed equivale tutto sommato a una arbitraria limitazione. Considerazioni analoghe, mutatis mutandis, fece HeIDEgGer in merito alla traduzione latina di physis con natura. Non si parla qui di errori di traduzione, ma più fondamentalmente del fatto che tradurre non è mai un atto innocente, neutrale, normalizzabile, scontato o, è necessario ribadirlo oggi con forza, automatico o comunque demandabile a un automa. Tradurre non è semplicemente una commutazione di codice, ma è trasposizione di un intero, cioè di un concetto e tutto l'universo mentale a cui pertiene e da cui non può essere rescisso, a un altro intero, cioè a un altro concetto a sua volta inserito e solidale di un altro universo concettuale.

[2] Sono queste alcune considerazioni nate durante l'elaborazione della versione italiana della Guida di legislazione, la cui pubblicazione all'inizio di quest'anno (Ufficio federale di giustizia, Guida di legislazione. Guida all'elaborazione degli atti normativi della Confederazione, $1^{\text {a }}$ edizione italiana, testo a cura della Divisione italiana dei Servizi linguistici centrali della Cancelleria federale, Berna 2019), è certo un lieto evento da salutare quale segno tangibile della presenza sempre più capillare della lingua italiana nell'apparato amministrativo federale, ma ha comportato grandi difficoltà riconducibili alla parziale inesistenza di quell'intero a cui abbiamo appena fatto riferimento.

[3] Un manuale di legistica a livello federale esiste da più di vent'anni e ha conosciuto già più edizioni (questa è la quarta), ma soltanto in versione tedesca e francese. Questo bilinguismo strumentale ha creato, e crea tuttora, una grave lacuna nel sostrato terminologico e concettuale in lingua italiana con serie ripercussioni sui lavori di approntamento del testo italiano di quest'opera di consultazione. Le difficoltà infatti sono state molteplici: scarsa competenza giuridica in lingua italiana presso l'ufficio responsabile, pochi testi dottrinali esistenti in Svizzera nella lingua di Dante con conseguente mancanza di riferimenti concettuali per campi specifici come ad esempio le norme primarie, i tributi, i dintorni dottrinali del Normkonzept o, ancora, le distinzioni nozionali e lessicali relative all'impatto della regolamentazione. La problematica non è nuova: l'assenza di funzionari di concetto italofoni marginalizza la lingua italiana dai lavori d'ideazione dei progetti provocando carenze terminologiche specialistiche che a loro volta pregiudicano - se non paralizzano - la redazione di testi di riferimento in lingua italiana, rendendo a loro volta difficile se non impossibile il lavoro in lingua italiana per i pochi giuristi italofoni pur presenti nell'amministrazione. Questa pubblicazione vorrebbe spezzare tale circolo vizioso e offrire uno strumento per pensare, elaborare, coordinare e affinare la legislazione anche in italiano, e questo fin dal principio dei lavori.

[4] È anche un modo per contribuire all'edificazione dell'universo concettuale, l'intero, di cui si diceva. Si consideri l'esempio della parola Normkonzept, di cui non esiste in italiano un equivalente consolidato e per la quale si è dovuto creare un nuovo termine. Grande sarebbe stata la tentazione di tradurre questa parola con il termine "concetto normativo». Prima di assegnare un nome a qualcosa, occorre però verificare cosa si intende. Il «concetto» è solitamente l'idea di qualche cosa, l'impostazione generale, che resta però confinata alla sfera astratta e concettuale appunto, mentre per Normkonzept si intende già una bozza (il francese parla infatti di «esquisse d'acte normatif»), uno schema del disposto normativo da elaborare, tanto più che nel Normkonzept va presentata ad esempio la struttura sommaria dell'atto, la densità normativa prevista e i 
contenuti principali (n. marg. 162 segg.). È quindi una sorta di archetipo concreto che mostra, fa vedere come già il "paradigma» platonico (Leggi, 692c), è una via di mezzo tra l'idea e la realizzazione finale, qualche cosa che nelle arti costruttive si chiama modello, sia nel senso propositivo e plastico di prima rappresentazione del progetto a venire (è la maquette francese), sia nell'accezione esemplare quale figurazione da seguire per la realizzazione vera e propria (quasi, dunque, un prototipo). Modello normativo dunque. È da notare che non vi è un equivalente nella procedura legislativa italiana, la quale conosce l'analoga, ma non identica, «relazione illustrativa».

[5] Ma, infine, la presente versione vuole essere anche un monito: ancora una volta è stato infatti confermato che impostando in modo plurilingue i lavori di redazione ab initio, come raccomandato del resto dalla Guida (n. marg. 87), si traggono grandi benefici sotto il profilo qualitativo, giacché la traduzione permette di individuare eventuali errori nel testo originale e di porvi quindi rimedio. Tradurre non è mai un atto innocente, ma non tradurre, potremmo concludere, comporta rischi ancora maggiori.

Jean-Luc Egger, Cancelleria federale, Servizi linguistici centrali, Divisione italiana, Berna, e-mail: jean-luc.egger@bk.admin.ch.

Ringrazio Tiziano Giabardo per la rilettura di queste righe e per il grande impegno intorno alla Guida di legislazione. 